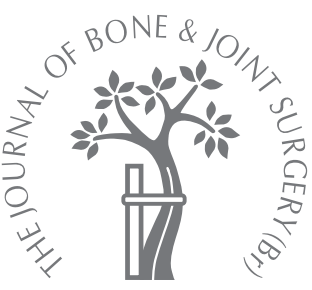

S. A. Hobson,

A. Karantana,

S. Dhar

From Queens

Medical Centre,

Nottingham,

England

\title{
Total ankle replacement in patients with significant pre-operative deformity of the hindfoot
}

We carried out 123 consecutive total ankle replacements in 111 patients with a mean followup of four years (2 to 8). Patients with a hindfoot deformity of up to $10^{\circ}$ (group $A, 91$ ankles) were compared with those with a deformity of $11^{\circ}$ to $30^{\circ}$ (group $B, 32$ ankles). There were 18 failures $(14.6 \%)$, with no significant difference in survival between groups $A$ and $B$. The clinical outcome as measured by the post-operative American Orthopaedic Foot and Ankle Surgeons score was significantly better in group $B(p=0.036)$. There was no difference between the groups regarding the post-operative range of movement and complications. Correction of the hindfoot deformity was achieved to within $5^{\circ}$ of neutral in 27 ankles (84\%) of group B patients. However, gross instability was the most common mode of failure in group B. This was not adequately corrected by reconstruction of the lateral ligament.

Total ankle replacement can safely be performed in patients with a hindfoot deformity of up to $30^{\circ}$. The importance of adequate correction of alignment and instability is highlighted.

Improvements in the design of total ankle replacement (TAR) has led to it becoming a viable alternative to fusion in the treatment of ankle arthrosis. The mobility provided by an ankle arthroplasty improves function and, it is suggested, leads to a reduction in the development of subsequent degenerative changes in the subtalar and midfoot joints, which often occur following fusion. ${ }^{1}$ The rates of revision and re-operation following TAR remain high relative to other types of joint arthroplasty, ${ }^{2}$ and therefore the clear benefits over fusion remain to be proved. ${ }^{3}$ Careful selection of the patients and tailoring of the treatment to the complex and varied deformities of the individual ankle may be the key to success. There is also a significant learning curve associated with the procedure. ${ }^{4}$

Some authors have suggested that preoperative deformity of the hindfoot should be a relative contraindication to ankle arthroplasty because of the risk of 'edge loading' of the insert and higher rates of failure. , $^{5,6}$

This study aims to identify the factors that influence a satisfactory outcome following TAR, and in particular, to determine whether significant pre-operative deformity of the hindfoot increases the rates of complication and failure. We also consider the degree of correction of the deformity that is achievable, and the procedures that can usefully be performed to address deformity and the resultant instability.

\section{Patients and Methods}

Between March 1999 and January 2006 a total of 123 consecutive Scandinavian Total Ankle Replacements (STAR) (Waldemar Link, Hamburg, Germany) were carried out in 111 patients by the senior author (SD). This device is an unconstrained, mobile-bearing prosthesis with a double coating of titanium and calcium phosphate and is inserted without cement. The mean follow-up was four years (2 to 8).

Before operation the coronal deformity at the tibiotalar joint was measured on standardised, weight-bearing anteroposterior radiographs of the ankles (Fig. 1). After ankle replacement this radiological measure cannot be used because the implant hides the body of the talus, so that direct measurement of its orientation cannot be made. Therefore, goniometric and radiological measurement of the position of the hindfoot as a whole was used to assess the post-operative alignment.

The patients were divided into two groups, depending upon the degree of their initial deformity. Group A had deformity of up to $10^{\circ}$ and group $\mathrm{B}>10^{\circ}$. Deformity in group B did not exceed $30^{\circ}$. The predominant deformity was varus (Fig. 2).

There were 91 replacements in group A and 32 in group B. There were five deaths overall in the original cohort, all in group $\mathrm{A}$, and all from causes unrelated to the arthroplasty. Three of these patients died within two years 


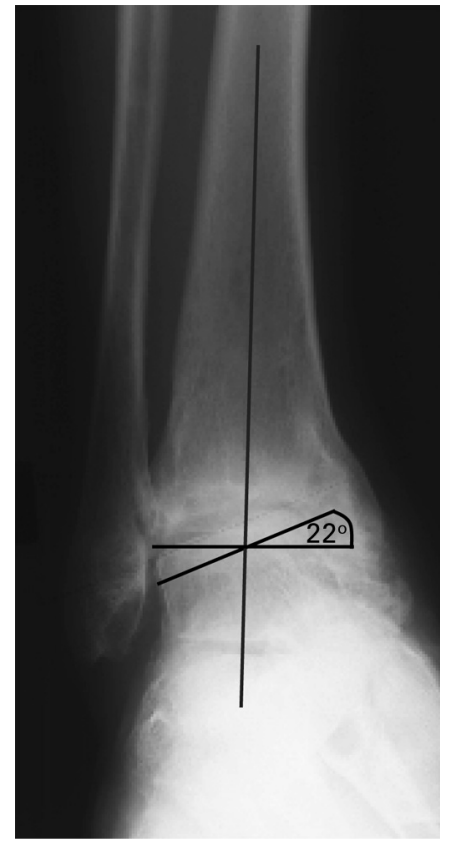

Fig. 1

Measurement of pre-operative coronal deformity (the angle between a line along the superior talar dome relative to a line perpendicular to the axis of the tibial shaft).

of operation and were not included in the analysis. No surviving patient was lost to follow-up. The mean age of the patients was 64 years ( 33 to 83 ) and the predominant disease process was osteoarthritis, with $53 \%$ (65 of 123) having no documented history of previous ankle trauma, $28 \%$ (35 of 123 ) with osteoarthritis secondary to previous injury, and $19 \%$ (23 of 123) with an inflammatory arthropathy (Table I). There were no statistically significant differences between the two groups with regard to age, gender or indication.

Operative technique and post-operative regime. The operation was performed through a standard anterior approach to the ankle via the bed of either the tendons of tibialis anterior or the extensor hallucis longus. A thorough debridement of the joint was carried out by excising the excess capsule, synovial tissue and the osteophytes. This frequently involved a thorough clearance of the medial and lateral gutters of all soft tissue and bony osteophytes. Often this resulted in 'releasing' the talus sufficiently to correct the deformity passively. The aim at this stage was to achieve a plantigrade foot with no residual varus, valgus or equinus. Inability to do so was usually a result of either severe extra-articular soft-tissue contracture, true bony deformity or both. It was necessary to avoid injury to the anterior talofibular ligament during this debridement. Care was also taken to avoid injury to the tendon of tibialis posterior, which was often visualised. In the initial years, residual

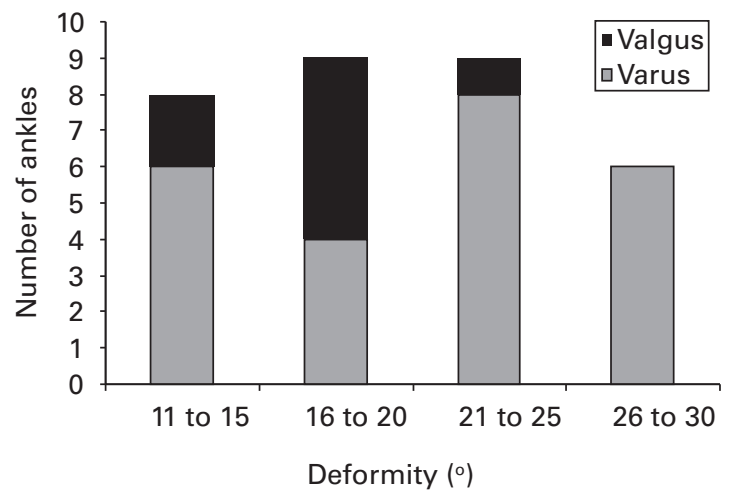

Fig. 2

Graph showing the distribution of deformity in group B.

deformities were accepted at this stage and once the implant had been inserted, correction of the hindfoot was obtained either by a calcaneal osteotomy or subtalar fusion, or both. These were carried out concomitantly or as staged procedures, depending on the complexity of the index operation. As experience with the procedure grew, in the event of persisting varus following a thorough intra-articular release of soft tissues, extra-articular releases were carried out. These included a lengthening of the tibialis posterior tendon and a sequential release of the deltoid ligament, which in four patients was completely transected. If further correction was required, bony debridement was carried out in the medial gutter to allow the talus to rotate into normal alignment. This often included removal of $1 \mathrm{~mm}$ of the distal tibia. Occasionally, despite these measures, a deformity persisted and was accepted, to be corrected by a separate hindfoot procedure.

Bony cuts were now made using the standard jigging systems to insert the prostheses without bone cement following thorough pulsed lavage. At this stage, stability was assessed, and if there was any doubt about the integrity of the lateral ligaments, reconstruction of these structures was carried out. If there was residual equinus percutaneous lengthening of the tendo-Achillis was required. At the end of the procedure a full plaster containing an A-V impulse slipper foot pump was applied and the pump maintained for 24 hours. Patients were discharged on average on the third day after operation. They were permitted to touch weight-bear in plaster for a period of six weeks, and then allowed to mobilise freely out of plaster.

Clinical evaluation. The patients were assessed at their most recent follow-up using the American Orthopedic Foot and Ankle Surgeons' (AOFAS) score. ${ }^{7}$ This was selected as the rating system most commonly used for studies of TAR. ${ }^{3}$ The alignment of the hindfoot on weight-bearing was also measured, as well as the arc of dorsiplantar flexion achieved at the tibiotalar joint. All measurements were made by a single assessor, using a goniometer. 
Table I. Demographic and clinical data

\begin{tabular}{llll}
\hline & & Group & \\
\cline { 2 - 4 } & All & A & B \\
\hline $\begin{array}{l}\text { Number of ankles } \\
\text { Mean age in yrs (range) }\end{array}$ & 123 & 91 & 32 \\
Gender (\%) & 64 (33 to 83) & 64 (33 to 81) & 66 (37 to 83) \\
$\quad$ Female & & & \\
$\quad$ Male & $39(32)$ & $32(35)$ & $8(25)$ \\
& $84(68)$ & $59(65)$ & $24(75)$ \\
Indications (\%) & & & \\
$\quad \begin{array}{l}\text { Osteoarthritis } \\
\text { Inflammatory joint disease }\end{array}$ & $23(19)$ & $17(19)$ & $6(19)$ \\
\hline
\end{tabular}

Radiological evaluation. Patients had annual clinical assessments together with standardised weight-bearing anteroposterior and lateral radiographs of the ankle. These were reviewed by a single observer for alignment of the prosthesis using the method described by Wood and Deakin, ${ }^{6}$ edge loading of the insert, or the presence of lucent lines or osteolysis about the tibial component. This allowed sequential comparison of the radiographs for migration or progressive osteolysis.

Statistical analysis. Survival analysis was carried out using the Kaplan-Meier survival curve, with failure of the prosthesis as the endpoint. We compared the survival curves for patients with and without significant pre-operative deformity using the non-parametric log-rank test. Regression analyses using the Cox proportional-hazards method were performed to determine whether the age at the time of the ankle arthroplasty, gender, and the indication for the operation had an influence on failure. Statistical significance was accepted if the p-value was $<0.05$.

The clinical outcome using the AOFAS score was compared between the two groups using a two-sample $t$-test for normal distributions. A retrospective analysis was performed to calculate power. This found the study to have $95 \%$ power to detect a $10 \%$ difference in the mean AOFAS scores (two-sided $\alpha 0.05$ ).

\section{Results}

Rates of failure. There were 18 failures (14.6\%). So far, two ankles have been revised to fusion and 13 have undergone revision of the components, five of the insert only. Three patients are awaiting revision, two to fusion and one for revision of components. There have been no additional radiological failures. Cox regression modelling showed that age, gender and the indication for arthroplasty had no impact on the risk of failure $(\mathrm{p}>0.05)$.

In group A there were 12 failures with six in group B. Survival curves for each group are shown in Figure 3 and the life table analysis for survival in Table II. We compared the survival curves for patients with and without significant pre-operative deformity using the non-parametric log-rank test at five and seven years. There was no significant difference between the two groups overall $(\mathrm{p}=0.752)$.
Mode of failure. This is shown for each group in Table III. In group A, the two cases of instability involved edge loading rather than gross instability. These required only exchange of the insert and correction of soft tissue or bony alignment. Both revision procedures were performed at two years post index operation. One insert required exchange for a fracture at four years. Two patients required exchange for subluxation of the insert noted on the immediate postoperative radiograph. One patient had a successful revision of the talar component for painful impingement. The most common cause of failure in group B was instability, with three of these five ankles demonstrating gross varus or valgus instability and subluxation, necessitating fusion. All but one of these ankles had a pre-operative deformity $\left(>20^{\circ}\right)$ in the coronal plane at the tibiotalar joint.

Clinical outcomes. The mean AOFAS score at the last follow-up in group A was 77 (SD 15.9) and in group B 86 (SD 10.1), a significant difference $(\mathrm{p}=0.036)$. The mean difference in scores was $9 \%$ (95\% confidence interval 1 to 17$)$, with patients in group B scoring significantly higher $(\mathrm{p}=0.036)$.

The post-operative arcs of movement were similar for the two groups with a mean arc of dorsiplantar flexion of $19.7^{\circ}$ $\left(0^{\circ}\right.$ to $\left.40^{\circ}\right)$ for group A and $18.7^{\circ}\left(5^{\circ}\right.$ to $\left.60^{\circ}\right)$ for group $\mathrm{B}$.

Radiological assessment. Seven ankles, all in group A, had lucent lines behind the tibial component which did not appear progressive. Four patients developed isolated cystic areas behind the tibial component or within the malleoli. One occurred in group B and healed spontaneously. In group A, one healed after grafting and two more are asymptomatic and under observation. No subsidence or migration of the components has been observed on sequential radiographs. No cases of edge loading have been seen in the surviving ankles.

Correction of deformity in group B. Alignment of the hindfoot to within $5^{\circ}$ of varus or valgus was achieved in 27 ankles $(84 \%)$, with a neutral hindfoot in one-third of the group. The majority of this correction was achieved through the standard bony cuts and soft-tissue releases at the primary procedure. Additional procedures for correction of deformity which were performed at operation 


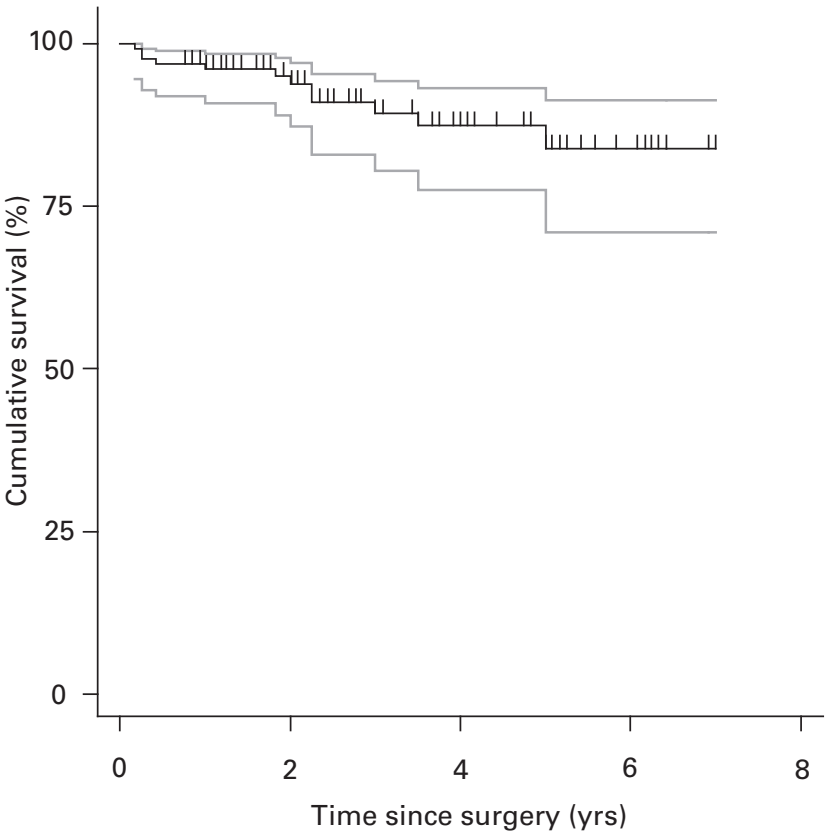

Fig. 3a

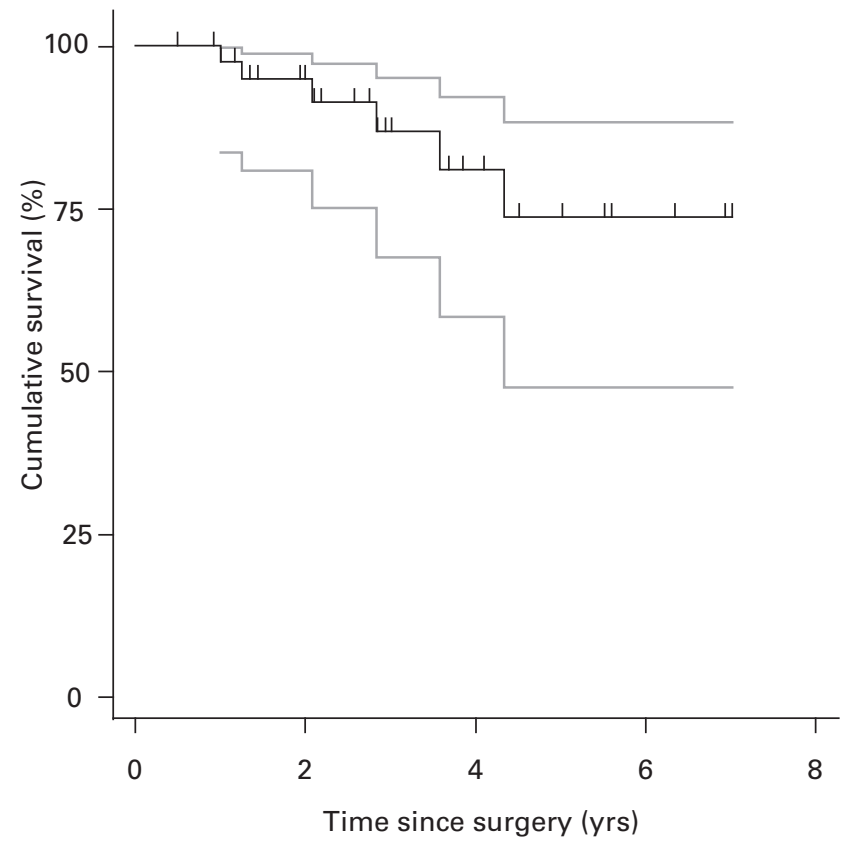

Fig. 3b

Kaplan-Meier estimated survival curve and $95 \%$ confidence interval for a) group A, and b) group B, with failure for any reason taken as the endpoint.

Table II. Life table for survival data in groups A and B

\begin{tabular}{|c|c|c|c|c|c|}
\hline Group & Years since operation & Number at start & Number revised & Survival rate (\%) & $95 \% \mathrm{Cl}^{*}$ \\
\hline \multirow[t]{7}{*}{$A$} & 0 to 1 & 91 & 4 & 96 & 89 to 98 \\
\hline & 1 to 2 & 87 & 2 & 93 & 86 to 97 \\
\hline & 2 to 3 & 81 & 3 & 89 & 80 to 94 \\
\hline & 3 to 4 & 55 & 2 & 86 & 75 to 92 \\
\hline & 4 to 5 & 39 & 0 & 86 & 75 to 92 \\
\hline & 5 to 6 & 25 & 1 & 81 & 66 to 90 \\
\hline & 6 to 7 & 12 & 0 & 81 & 66 to 90 \\
\hline \multirow[t]{8}{*}{ B } & 0 to 1 & 32 & 0 & 100 & - \\
\hline & 1 to 2 & 32 & 1 & 97 & 79 to 99 \\
\hline & 2 to 3 & 29 & 2 & 89 & 68 to 96 \\
\hline & 3 to 4 & 17 & 1 & 83 & 59 to 93 \\
\hline & 4 to 5 & 12 & 1 & 75 & 48 to 89 \\
\hline & 5 to 6 & 9 & 0 & 75 & 48 to 89 \\
\hline & 6 to 7 & 5 & 0 & 75 & 48 to 89 \\
\hline & 7 to 8 & 3 & 1 & 38 & 2 to 79 \\
\hline
\end{tabular}

* $95 \% \mathrm{Cl}, 95 \%$ confidence interval

prior to or following the index procedure are shown in Table IV. Calcaneal osteotomy was undertaken in six ankles, with good results for symptomatic deformity and only one failure. Partial deep deltoid release, with or without lengthening of tibialis posterior, was required to achieve a neutral hindfoot at operation in four patients. Frank instability was present in three ankles following replacement which required reconstruction of the lateral ligament as a subsequent procedure. All were unsuccessful.
Complications. Delayed wound healing, including superficial infection, occurred in ten patients from group A and two from group B. All of these subsequently healed. There were no cases of deep infection.

There were three cases of heterotopic ossification. None required operative intervention. No cases of venous thromboembolism occurred. There were 13 fractures, distributed in a similar manner among the two groups (Table $\mathrm{V})$. The numbers are not large enough to perform a formal statistical comparison. The incidence of intra- and 
Table III. Mode of failure by group

\begin{tabular}{llllll}
\hline & Group A & & \multicolumn{2}{l}{ Group B } \\
\cline { 2 - 3 } \cline { 5 - 6 } Mode of failure & Number & \% of all failures & Number & \% of all failures \\
\hline Instability & 2 & 11 & 5 & 0 & 0 \\
Fracture & 4 & 22 & 0 & 0 & 0 \\
Fracture/dislocation of the insert & 3 & 6 & 0 & 0 \\
Component malposition & 1 & 6 & 1 & 6 \\
Aseptic loosening & 1 & 6 & &
\end{tabular}

Table IV. Additional procedures to address deformity/instability in successful and failed ankles in group B

\begin{tabular}{llll}
\hline & Procedure & Success & Failure \\
\hline Pre-operatively & Calcaneal osteotomy & 1 & 0 \\
& Subtalar fusion & 1 & 0 \\
\multirow{2}{*}{ Intra-operatively } & Deltoid release & 2 & 1 \\
& Tibialis posterior tendon lengthening and deltoid release & 0 & 1 \\
& Lateral ligament reconstruction & 1 & 0 \\
Post-operatively & & 3 & 1 \\
& Calcaneal osteotomy & 0 & 3 \\
\hline
\end{tabular}

Table V. Fractures by group

\begin{tabular}{clll}
\hline & Fractures (\%) & Intra-operative fracture & Post-operative stress fracture \\
\hline Group & & & \\
A & $9(10)$ & 3 & 6 \\
B & $4(12.5)$ & 1 & 3 \\
\hline
\end{tabular}

post-operative fracture has been reducing over the course of the study.

\section{Discussion}

Patients with a pre-operative deformity of the hindfoot of up to $30^{\circ}$ did not have an increased risk of failure, complications or adverse clinical outcomes compared with those with neutral alignment of the hindfoot if adequate steps were taken at operation to achieve neutral alignment and stability. The mode of failure in this group was more likely to be instability, especially with deformities $>20^{\circ}$.

Comparing our results with those of other series is difficult, as patient selection, the indications and the choice of revision procedure vary considerably. The rates of survival at five years for the semi-constrained Agility prosthesis range (DePuy Orthopaedics Ltd, Warsaw, Indiana) from $80 \%$ to $95 \%,{ }^{2,7}$ for the Buechel-Pappas prosthesis (Endotec, South Orange, New Jersey) $79 \%$ to $90 \%, 5$ and for the STAR $70 \%$ to $92.7 \% .{ }^{6,9,10}$ A recent metaanalysis quoted a five-year survival of $78 \%$ for ankle arthroplasty. ${ }^{11}$ Our survival rates of $86 \%$ and $75 \%$ are comparable. In contrast to the quoted series, few of our failures required revision to an arthrodesis, most requiring only revision of components.
Some studies have found age $^{2}$ and inflammatory arthropathy ${ }^{11}$ to be associated with lower rates of failure. However, we have found no increased risk of failure dependent on age, indication or gender.

Contrary to our findings, Doets et $\mathrm{al}^{5}$ noted that patients with a pre-operative deformity $>10^{\circ}$ had an increased rate of failure. However, their number of such patients was relatively small, and additional procedures to address correction of deformity were not noted to have been performed.

Wood and Deakin ${ }^{6}$ and Haskell and Mann ${ }^{12}$ found a higher rate of edge loading in patients with a pre-operative deformity of the hindfoot. We found that significant instability is more common in the group with deformity, which suggests that adequate correction of deformity may prevent failure due to instability. Our lower rates of failure in these patients may be due to our higher rate of operative correction. In many cases this was achieved without additional procedures such as soft-tissue release and calcaneal osteotomy or subtalar fusion, although these were performed intra-operatively where necessary. It is safe to perform realignment procedures at the time of primary ankle replacement, without increasing the rates of complications. ${ }^{13}$ Haskell and Mann ${ }^{12}$ have demonstrated lasting shortterm correction of hindfoot deformity with TAR. Our study shows that this correction is maintained in the longer term. 
One potential caution in the interpretation of our results is the quantification of hindfoot deformity. We compared the pre-operative radiological measurement of deformity with both the goniometric and the radiological deformity post-operatively. The clinical measurements quoted are at increments of $5^{\circ}$, and accuracy to this level using a goniometer could be challenged. These measurements also reflect deformity at both the tibiotalar and the subtalar joints. The radiological measurement is purely tibiotalar. Accurate radiological quantification of subtalar deformity is difficult. We aimed to achieve a clinically neutral, balanced hindfoot, and this is reflected in our results.

We have not separated the results according to whether the pre-operative deformity was in varus or valgus. There were a smaller number of valgus ankles (eight), which would be unlikely to produce statistically useful conclusions for that group. The general principles of restoring soft-tissue balance around an arthroplasty applies regardless of the direction of the deformity.

The clinical outcomes in terms of the American Orthopedic Foot and Ankle Surgeons' score compared favourably with those of previous studies. ${ }^{3}$ None had specifically compared outcomes between patients with and without a significant pre-operative deformity of the hindfoot. The higher scores in the group with significant deformity may be representative of their having more to gain from the procedure. Our rates of loosening and the incidence of component migration requiring revision are lower than those quoted by other studies. ${ }^{5,6}$ This may be explained by the superior bone-implant interface achieved by the dual titaniumcalcium phosphate coating on the prosthesis, which was not used on older designs. ${ }^{6,14}$ As expansile osteolysis has been noted to appear from a mean of 35 months, ${ }^{7}$ more loosening or lysis may become evident over a longer period of followup. However, $45 \%$ of our ankles had a follow-up in excess of this period, with no evidence of increased loosening.

Our results demonstrate that once failure due to gross instability has occurred, it cannot be addressed adequately by lateral ligament reconstruction alone. It is necessary to correct deformity at an early stage by a combination of soft-tissue releases and bony procedures, as this mode of failure is difficult to salvage once it has occurred. We currently plan an ankle replacement followed by realignment surgery as a second stage if required. We did not perform ankle replacements in patients with deformity $>30^{\circ}$, as we felt that sufficient correction could not be achieved in these cases, and therefore arthrodesis was our preferred option.

Complications in our study were low in terms of wound healing, infection and heterotopic ossification. ${ }^{6,15}$ Rates of fracture in TAR have been noted to be $20 \% .{ }^{16}$ Our rates were significantly lower and are improving with time, suggesting that they are related to a learning curve with the technique. ${ }^{6,13}$ Both ankle and hindfoot fusion have previously been shown to be a safe and effective salvage procedure after failed TAR, ${ }^{17,18}$ and our patients who underwent ankle fusion for failure due to instability had satisfactory results.

Patients with a hindfoot deformity of up to $30^{\circ}$ can safely undergo TAR, expecting to achieve equivalent results to those of patients with neutral hindfoot alignment. However, careful attention should be paid to correction of alignment to prevent failures due to instability, which may be more difficult to achieve in ankles with higher degrees of pre-operative deformity.

The authors wish to thank K. Lee, Extended Scope Practitioner Physiotherapist, for her role in this study as assessor.

No benefits in any form have been received or will be received from a commercial party related directly or indirectly to the subject of this article.

\section{References}

1. SooHoo NF, Zingmond DS, Ko CY. Comparison of reoperation rates following ankle arthrodesis and total ankle arthroplasty. J Bone Joint Surg [Am]2007;89-A:2143-9.

2. Spirt AA, Assal M, Hansen ST Jr. Complications and failure after total ankle arthroplasty. J Bone Joint Surg [Am] 2004;86-A:1172-8.

3. Haddad SL, Coetzee JC, Estok R, et al. Intermediate and long-term outcomes of total ankle arthroplasty and ankle arthrodesis: a systematic review of the literature. $J$ Bone Joint Surg [Am] 2007;89-A:1899-905.

4. Kumar A, Dhar S. Total ankle replacement: early results during learning period. Foot Ankle Surg 2007;13:19-23.

5. Doets HC, Brand R, Nelissen RG. Total ankle arthroplasty in inflammatory joint disease with use of two mobile-bearing designs. J Bone Joint Surg [Am] 2006;88A:1272-84

6. Wood PL, Deakin S. Total ankle replacement: the results in 200 ankles. J Bone Joint Surg [Br] 2003;85-B:334-41.

7. Knecht SI, Estin M, Callaghan JJ, et al. The agility total ankle arthroplasty: seven to sixteen-year follow-up. J Bone Joint Surg [Am] 2004;86-A:1161-71.

8. Wood PL, Sutton C, Mishra V, Suneja R. A randomised, controlled trial of two mobile-bearing total ankle replacements. J Bone Joint Surg [Br] 2009;91-B:69-74.

9. Wood PL, Prem H, Sutton C. Total ankle replacement: medium-term results in 2000 Scandinavian total ankle replacements. J Bone Joint Surg [Br] 2008;90-B:605-9.

10. Anderson T, Montgomery F, Carlsson A. Uncemented STAR total ankle prostheses: three to eight-year follow-up of fifty-one consecutive ankles. J Bone Joint Surg [Am] 2003;85-A:1321-9.

11. Hurowitz EJ, Gould JS, Fleisig GS, Fowler R. Outcome analysis of agility total ankle replacement with prior adjunctive procedures: two to six year follow-up. Foot Ankle Int 2007;28:308-12.

12. Haskell A, Mann RA. Ankle arthroplasty with preoperative coronal plane deformity: short term results. Clin Orthop 2004;424:98-103.

13. Schuberth JM, Patel S, Zarutsky E. Peri-operative complications of the agility total ankle replacement in 50 initial, consecutive cases. J Foot Ankle Surg 2006;45:139-46.

14. Carlsson A, Markusson P, Sundberg M. Radiostereometric analysis of the doublecoated STAR total ankle prosthesis: a 3-5 year follow-up of 5 cases with rheumatoid arthritis and 5 cases with osteoarthrosis. Acta Orthopaedica 2005;76:573-9.

15. Valderrabano V, Hintermann B, Dick W. Scandinavian total ankle replacement: a 3.7 year follow up of 65 patients. Clin Orthop 2004;424:47-56

16. McGarvey WC, Clanton TO, Lunz D. Malleolar fracture after total ankle arthroplasty: a comparison of two designs. Clin Orthop 2004;424:104-10.

17. Culpan P, Le Strat V, Piriou P, Judet T. Arthrodesis after failed total ankle replacement. J Bone Joint Surg [Br] 2007;89-B:1178-83.

18. Kotnis R, Pasapula C, Anwar F, Cooke PH, Sharp RJ. The management of failed ankle replacement. J Bone Joint Surg [Br]2006;88-B:1039-47. 